\title{
An artificial sphincter using shape memory alloy actuators
}

\author{
Toshiyuki TAKAGI, Yun LUO and Shinya HARA \\ Institute of Fluid Science, Tohoku University, Katahira 2-1-1, Aoba-ku, Sendai 980-8577, Japan \\ Tel/Fax: +81-22-217-5248 E-mail: takagi@ifs.tohoku.ac.jp \\ Tomoyuki YAMABE \\ Institute of Development, Aging and Cancer, Tohoku University \\ Shintaro AMAE and Motoki WADA \\ Graduate School of Medicine, Tohoku University \\ Hirokazu NAKAMURA \\ Sharp Co. Ltd. \\ (Received 14, July 2000 Accepted 30, November 2000)
}

\begin{abstract}
This paper describes the development of an artificial sphincter using shape memory alloy (SMA). With the artificial sphincter it is expected to enable the active bowel movement of patients with the lack of anal sphincters due to inborn intestinal atresia or medical operations of an artificial anus. The SMA actuator has the function of a sphincter muscle; keeping the artificial anus closed at the body temperature and opening it by heating. Investigations on the thermomechanical properties of the actuator have been carried out. Animal experiments have also been done, and the results showed potentially applicability of the artificial sphincter for implantation in living bodies.
\end{abstract}

Keywords: Shape memory alloys, actuators, thermomechanical properties, artificial sphincter, fecal incontinence.

\section{INTRODUCTION}

The introduction of intelligent actuators into various medical devices can not only reduce the risk in medical operations, but also decrease the affliction of patients, therefore becomes an important issue in recent medical research. The continuously increasing needs of such intelligent medical systems require developments of new actuators in aspects of enhancing the quality of life. One notable example of such requirements comes from the medical treatment for patients with the fecal incontinence due to the colostomy, congenitally anorectal malformation or surgical operations for anorectal diseases. The lack of the anal sphincter is the main reason of the problem. So far, neosphincters using skeletal muscles, pressure regulatory balloon have been developed. However problems of fatigue limited their uses. The purpose of this paper is to develop an artificial sphincter using a shape memory alloy (SMA) actuator as one of the solutions of above problems.

SMAs have attracted increasing interest in engineering field since their discovery at the beginning of 1960s. The particular properties of 
SMAs; the shape memory effect (SME) and the pseudoelasticity are proposed to be used in thermomechanical sensors, actuators and other intelligent systems. ${ }^{1}$ SMA materials have the features of highly adaptive and high force-weight ratio. The great advantage of using SMAs in sensor and actuator systems is that they can be operated actively through the thermomechanical energy conversion, therefore lead to simple designs. ${ }^{2,3}$ For medical applications, SMA materials have been applied in orthopoedic implants, ${ }^{4}$ contractile artificial muscles for an artificial heart, ${ }^{5}$ and a urethra valve. ${ }^{6}$ In this paper, the fundamental functions of the SMA artificial sphincter have been evaluated through the investigations of its thermomechanical properties, transient responses. The animal experiments for confirming the biocompatibility of the actuator have been conducted as well.

\section{MECHANISM OF ARTIFICIAL SPHINCTER}

The schematic drawing of the proposed artificial sphincter is illustrated in Fig. 1. The actuator consists of two SMA plates jointed by two hinges, and heating coils attached on the SMA plates. The length of the SMA plates is $70 \mathrm{~mm}$. The width and the thickness are $18 \mathrm{~mm}$ and $0.7 \mathrm{~mm}$, respectively. The material used for SMA plates; Ti51at\% Ni is known to exhibit all-round shape memory effect (ARSME), i.e. shows a shape change reverse to the "memorized" one in its martensitic phase. The transition temperatures of the SMA plates subjected to heat treatments $\left(400^{\circ} \mathrm{C}, 100 \mathrm{hr}\right.$ for annealing followed by $850^{\circ} \mathrm{C}, 20 \mathrm{~min}$. for the solution

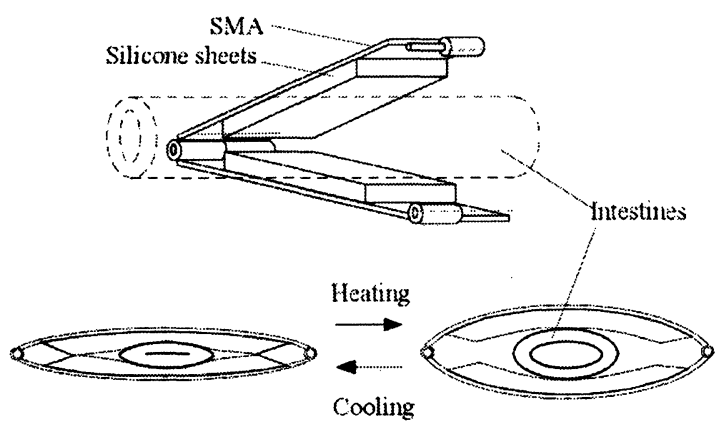

Fig. 1. Schematic drawing of the artificial sphincter using SMA. treatment) were evaluated by Differential Scanning Calorimetry (DSC). The entire temperature range for the ARSME is from $-5^{\circ} \mathrm{C}$ to $55^{\circ} \mathrm{C}$. Since the actuator is assumed to be used in a temperature range near human body temperature (around $37^{\circ} \mathrm{C}$ ). Therefore only the transformations between the austenite phase (A-phase) and the rhombohedral phase (R-phase) need to be considered. The start and finish temperatures are obtained by DSC tests as $\mathrm{A}_{\mathrm{s}}=39^{\circ} \mathrm{C}, \mathrm{A}_{\mathrm{f}}=55^{\circ} \mathrm{C}$ for A-phase and $\mathrm{M}_{\mathrm{s}}^{\prime}=43^{\circ} \mathrm{C}$, $\mathbf{M}_{\mathrm{f}}^{\prime}=35^{\circ} \mathrm{C}$ for $\mathrm{R}$-phase. The hysteresis of the transformation temperatures is around $16^{\circ} \mathrm{C}$. Typically the Young's modulus of SMA materials in the martensitic phase is $20 \%$ of that in the austenitic phase. ${ }^{7}$ This is the reason that most conventional SMA actuators require a bias spring to assist the shape recovery after an austenitic transition. In SMAs with ARSME, it was reported that the obtainable recovery forces in the R-phase is approximately half of that in the A-phase. ${ }^{8}$ Therefore, SMAs in R-phase might play a role of spring by itself, leading to a simple design of the SMA actuators. For heating, coils made of copper wires with insulating coatings were installed on the outer sides of SMA plates. Since the highest temperature for the complete reverse transformation might reach $55^{\circ} \mathrm{C}$, thermal insulating materials; cork sheets and sponge rubber sheets are covered at the outer and inner sides of the SMA plates respectively. The thermal insulating sheets prevent the surface temperature of the actuator from being higher than $40^{\circ} \mathrm{C}$; a critical temperature of cytoclasis for living bodies. The actuator could be installed around intestines, keeping it closed at body temperature by elastic forces of the SMA plates. When the electric power is applied to the coils for heating, the reverse transformation occurs in the SMA plates, accompanying by the shape changes from a flat shape to an arc, i.e. the restrained shape during annealing. The shape change results in a gap between two SMA plates for opening the intestines. After switching off the electric power, the shape of the SMA plates is recovered by natural cooling, and the intestines will be closed again. 


\section{THERMOMECHANICAL PROPERTIES}

\section{A. Shape changes under a thermal cycle}

The shape memory effect (SME) of the SMA plates has been demonstrated first. The shape change of the SMA plates is typically evaluated by the variation of their curvatures. Here, we focus on the obtainable maximum gap between the middle points of two deformed SMA plates, which should be equivalent to the diameter of intestines. The gap was evaluated by measuring the strain on the surface of the SMA plates. The temperature dependence of the gap is presented in Fig. 2. As seen in Fig. 2, the gap increases slightly from the room temperature of $29^{\circ} \mathrm{C}$ due to the local transformation, and exhibits a sharp rise from $39^{\circ} \mathrm{C}$ corresponding to the start temperature of the reverse transformation $A_{s}$. The increase of the gap saturates at the temperature of $55^{\circ} \mathrm{C} ; \mathrm{A}_{\mathrm{f}}$. The gap change in cooling corresponds also to the start and finish temperatures of the transformation from Aphase to R-phase. A maximum gap of $33.2 \mathrm{~mm}$ was obtained at $\mathrm{A}_{\mathrm{f}}$. Considering the initial gap due to the thickness of the silicone rubbers is $19 \mathrm{~mm}$, the increase of the gap is enough for opening the intestines with an average outer diameter of $15 \mathrm{~mm}$.

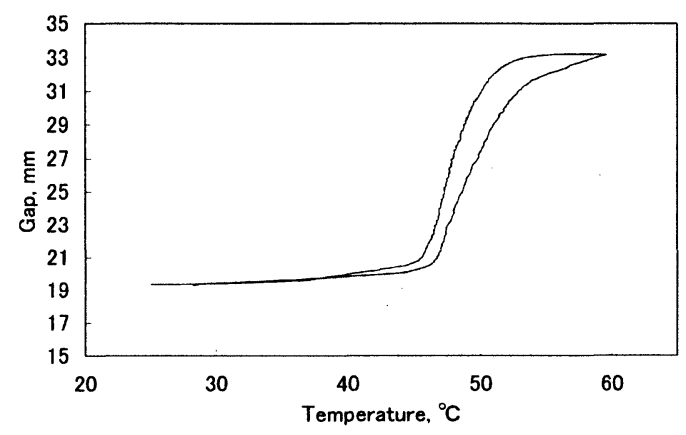

Fig. 2. Temperature dependence of the gap between two SMA plates in one thermal cycle (lower part for heating and upper part for cooling).

\section{B. Transient response of the artificial sphincter}

The transient responses of the artificial sphincter have been evaluated with complete thermal cycles. The investigations have been conducted on the time dependence of the temperature, the gap between two SMA plates, and the flow rate in intestines. For the confirmation of the closing and opening functions of the actuator, a hydrostatic pressure test has been carried out. The experimental setup illustrated in Fig. 3 consists of a water tank with float-feed for keeping a hydrostatic pressure, a thin silicone tube connected to a real intestine of pigs, and a flow meter. The artificial sphincter is installed around the intestine. At room temperature, the artificial sphincter exhibits a good closing function against a hydrostatic pressure of $65 \mathrm{mmHg}$, while the average inner pressure of intestines is estimated to be less than $50 \mathrm{mmHg}$ for human bodies. To determine the value of the input electric power, preliminary tests have been carried out for heating the SMA plates from $28^{\circ} \mathrm{C}$ to $60^{\circ} \mathrm{C}$ within 80 seconds. Based on the results of the tests, electric power of $16 \mathrm{~W}(2 \mathrm{~A}, 8 \mathrm{~V})$ was applied to the actuator for 80 seconds and then switched off. The temperature of the SMA plates was measured by thermocouples sandwiched between SMA plates and silicone sheets, while the gap between two SMA plates is calculated from the strain measured on the surface of the SMA plates. As seen in Fig. 4, the SMA plates are heated up to $55^{\circ} \mathrm{C}$ within one minute. Slight variations of the temperature change rate appear in both of the heating and cooling processes, which are attributed to the latent heat

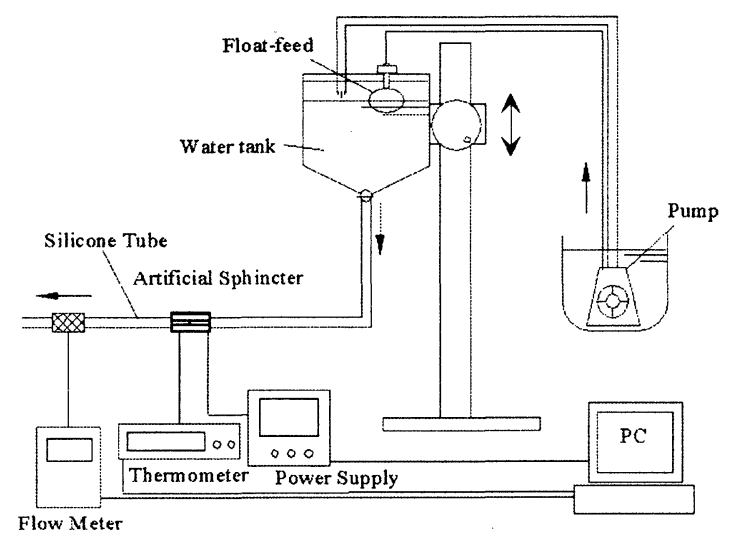

Fig. 3. Experimental setup for examining the opening-closing functions of the actuator under hydrostatic pressures. 


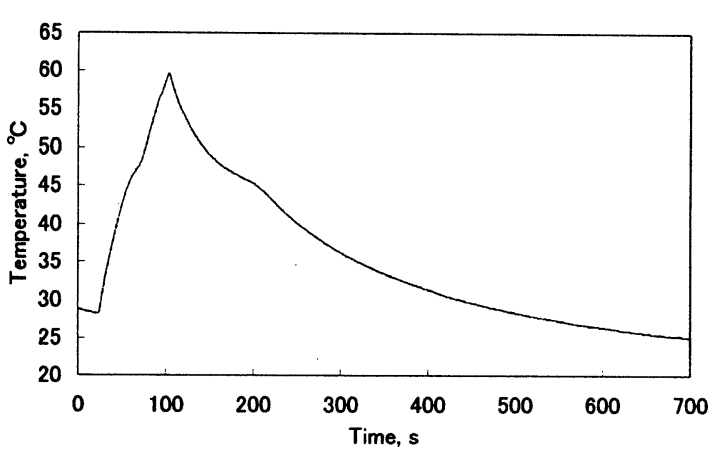

Fig. 4. Transient response of the temperature of the SMA plates.

effect during phase transformations. The gap shows a drastic increase by the heating and reaches a peak value of $33.2 \mathrm{~mm}$ (see Fig. 5). The flow rate exhibited a rise of $13 \mathrm{ml} / \mathrm{s}$ and was prevented with the decreased temperature and/or the gap (see Fig. 6). The temperature dependence of the flow rate in the complete thermal cycle is shown in Fig. 7. The flow rate shows drastic changes between $30^{\circ} \mathrm{C}$ and $45^{\circ} \mathrm{C}$ in both of the heating (lower curve) and cooling (upper curve) with small hysteresis.

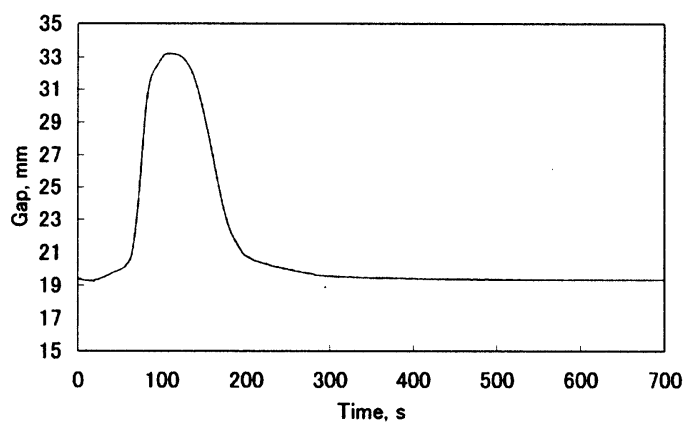

Fig. 5. Transient response of the gap.

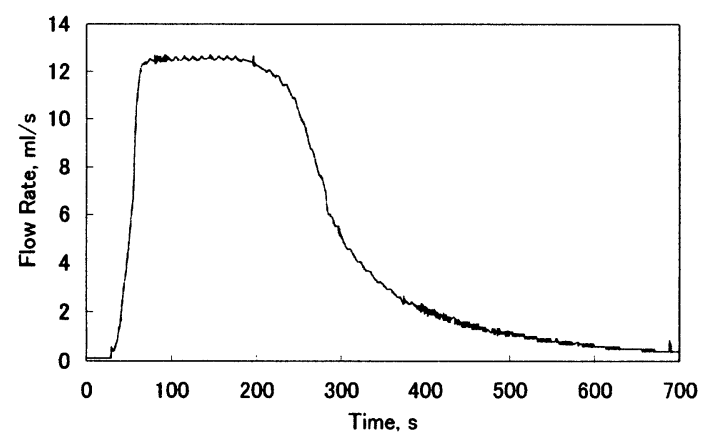

Fig. 6. Transient response of the flow rate.

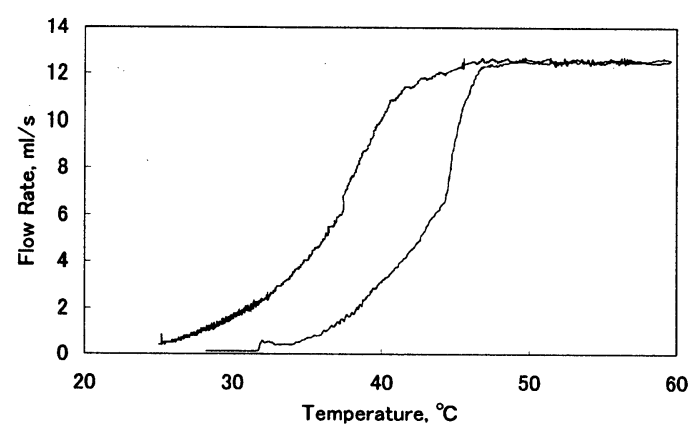

Fig. 7. Hysteresis of the flow rate during a heating-cooling cycle.

\section{ANIMAL EXPERIMENTS}

Animal experiments have been conducted for examining the fundamental functions and the biocompatibility of the actuator. The experiments were carried out on a pig with the same dimension of intestines as that of human body.

The preliminary test of the fundamental functions of the actuator was first conducted. As illustrated in Fig. 8, an artificial anus was made at the abdomen of the pig using a rectum after the resection operation of its colons. Then the artificial sphincter was installed around the rectum located between the abdominal wall and the peritoneum (see Fig. 9). The inside pressure of the rectum generated by the artificial sphincter was measured first. A pressure sensor was inserted from the artificial anus into the intestine and then moved out. The measured result of the pressure exhibits a rise of $50 \mathrm{mmHg}$ in the region clipped by the actuator, corresponding to the width of the SMA plates; $18 \mathrm{~mm}$ (see Fig. 10). The pressure tests have also been carried out with the

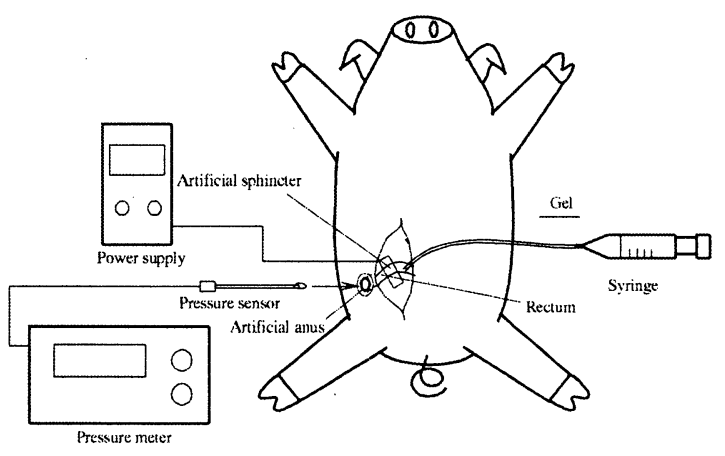

Fig. 8. A photograph of the artificial sphincter installed on the rectum of a pig. 


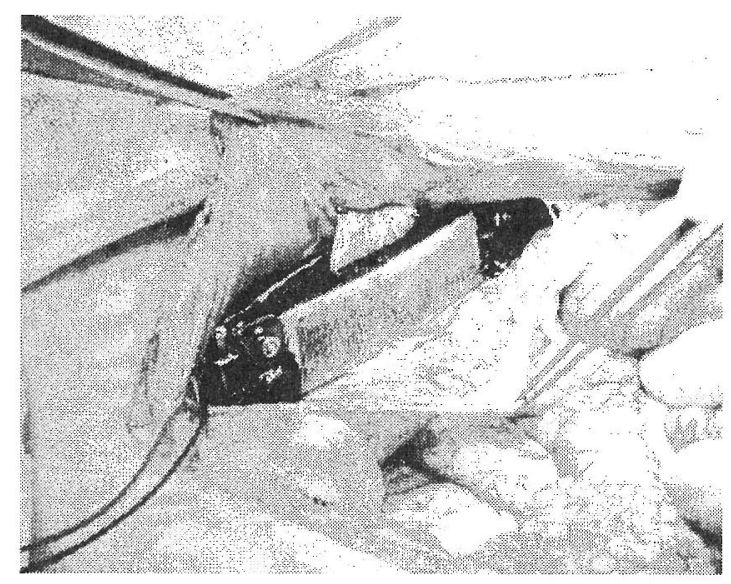

Fig. 9. A photograph of the artificial sphincter installed on the rectum of a pig.

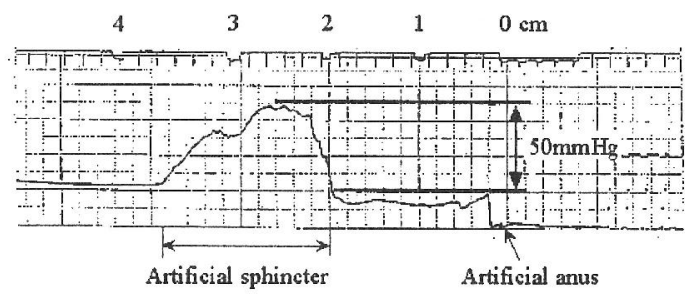

Fig. 10. Pressure distribution in an intestine clipped by the artificial sphincter.

inner part of the rectum subjected to a pressure. The inner pressure of the rectum was generated by pouring gel into the intestines. Figure 11 shows the measured pressure change during the test. As seen in Fig. 11, the pressure was increased up to $75 \mathrm{mmHg}$ without any leak of the gel, and then decreased with the opening of artificial sphincter by applying electric power. The discharge of the gel from the artificial anus was observed.

A clinical test on a living pig has also been carried out. The artificial sphincter enables the controlled bowl movement of the pig. From the dissecting examination after six days experiments, neither infections nor scars of a burn were observed

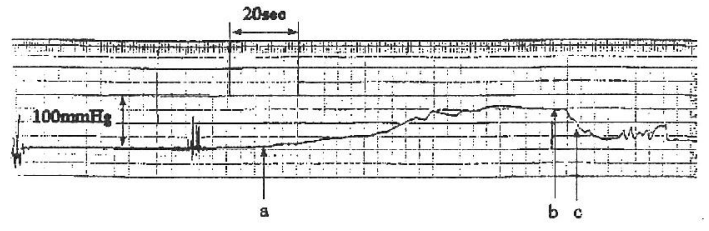

Fig. 11. Pressure change in the rectum (a: start pouring gel into the rectum, b: switch on the input power, c: discharge of gel was observed). in the body around the artificial sphincter Furthermore, the artificial anus exhibited a good condition suggesting the pressure due to the artificial sphincter was allowable. Additionally, in above experiments, the electric power was applied through a pair of current lead wires penetrated the body. The use of an inductive power transmission for the power supplement of the actuator could lead to a complete implantation-type artificial sphincter in further research.

\section{Conclusions}

In summary, an SMA artificial sphincter has been developed. The use of Ti51at\% Ni enables a simple design of the SMA actuator without a bias spring. Fundamentally thermomechanical behaviors of the actuator have been investigated experimentally. The developed artificial sphincter exhibits good closing function under the inner pressure of $75 \mathrm{mmHg}$. The animal experiments and clinical tests have also been conducted on a pig with similar dimensions of rectum with human body. The good biocompatibility of the artificial sphincter has been observed. The introduction of an inductive power transmission could enable the complete implantation of the artificial sphincter in further research.

\section{Acknowledgment}

Authors are grateful to Mr. Kiyoshi Yamauchi of Tokin Co. for providing SMA materials used in this work.

\section{REFERENCES}

1. C. M. Wayman, Some applications of shape memory alloys, Journal of metals, 6 (1990) pp.149-156

2. T. Takagi, J. Tani, S. Suzuki and M. Matsumoto, Dynamic Thermomechanical Response of an SMA Plate using an All-round Shape Memory Effect and an Application to an Adaptive FluidFlow Controller, Journal of Society of Mechanical Engineering, Vol. 63, 615 (199711) pp.3759-3765, in Japanese.

3. T. Takagi, J. Tani, S. Suzuki and M. Matsumoto, 
Evaluation of Electromagnetic, Thermal, Mechanical Properties of an All-round Shape Memory Alloy Plate, Journal of Society of Mechanical Engineering, Vol. 64, 624 (1998) pp.2966-2973, in Japanese.

4. J.L. Hughes, Evaluation of Nitinol for use as a material in the construction of orthopoedic implants, Final report DAMP 17-74-C-4041, (1977)

5. P.N. Sawyer, M. page, L. Beseliust, C. McCool, E. Lester, B. Stanczewski, S. Srinivasan, N. Ramasamy, Further study of nitinol wire as contractile artificial muscle for an artificial heart, Cardiov Diseases, Bull. Texas Heart Inst,
Vol. 3, No.1, (1976) pp.65-78

6. S. Chonan, Z. W. Jian, J. Tani, S. Orikasa, Y. Tanahashi, T. Takagi, M. Tanaka and J. Tanikawa, Development of an artificial urethral valve using SMA actuators, Smart Mater. Struct. 6 (1997) pp.410-414

7. K. Takezawa, S. Edo, S. Sato, Proc. ICOMAT79, Boston (1979), 661

8. M. Nishida and T. Honma, All-Round Shape Memory Effect (ARSME) in Ti-51at\% Ni Generated by Constrained Aging, Report of Research Institute of Mineral Ddressing and Metallurgy, Tohoku Univ., Japan, Vol.38, 2 (1982) pp.75-84, in Japanese.

Presented at 3rd Japan France Seminar on IMS 